\title{
Metabolic determination of decursinol using human liver microsome
}

\author{
Jiye Gu1, Young Jin Park¹, Chaeyeong Ahn¹, Dayoung Heo¹, Yuseong Chung ${ }^{1}$, \\ Kang Min Kim², Jae Seon Kang ${ }^{1}$, Jaesung Pyo ${ }^{1 *}$ \\ ${ }^{1}$ College of Pharmacy, ${ }^{2}$ Department of Pharmaceutical Science and Technology, Kyungsung University, Busan 48434, Republic \\ of Korea \\ *For correspondence: Email: jspyo@ks.ac.kr; Tel: +82 516634881
}

Sent for review: 18 July 2020

Revised accepted: 16 May 2021

\begin{abstract}
Purpose: To determine new metabolites of the main components of Angelica gigas known to give antiinflammation and pain relief

Methods: Decursinol and blank sample were metabolized in human liver microsomes. The metabolized samples were centrifuged and deproteinated by adding $3 \mathrm{~mL}$ acetonitrile. The acetonitrile layer was concentrated and reconstituted in methanol. Finally, the prepared sample was injected into the LC-QTOF-MS.

Results: Four new metabolites of decursinol with $\mathrm{m} / \mathrm{z}$ ranging from 263.0912 263.0920 were identified as hydroxylated forms of decursinol, and the hydroxylated position of each metabolite was characterized using TOF mass spectrum. Their error values of detected $\mathrm{m} / \mathrm{z}$ were $0.38 \sim 2.29 \mathrm{ppm}$, which indicates high accuracy of analysis.

Conclusion: Previously unreported decursinol metabolites have been identified in this study. The findings provide athe basis for further pharmaceutical studies and functional food development using decursinol.
\end{abstract}

Keywords: Angelica gigas, Decursinol, Metabolites, LC-Q-TOF-MS, Human liver microsome

\begin{abstract}
This is an Open Access article that uses a fund-ing model which does not charge readers or their institutions for access and distributed under the terms of the Creative Commons Attribution License (http://creativecommons.org/licenses/by/4.0) and the Budapest Open Access Initiative (http://www.budapestopenaccessinitiative.org/read), which permit unrestricted use, distribution, and reproduction in any medium, provided the original work is properly credited.

Tropical Journal of Pharmaceutical Research is indexed by Science Citation Index (SciSearch), Scopus, International Pharmaceutical Abstract, Chemical Abstracts, Embase, Index Copernicus, EBSCO, African Index Medicus, JournalSeek, Journal Citation Reports/Science Edition, Directory of Open Access Journals (DOAJ), African Journal Online, Bioline International, Open-J-Gate and Pharmacy Abstracts
\end{abstract}

\section{INTRODUCTION}

Angelica, a plant belonging to the genus Angelica and the family Apiaceae, has been widely used in Asia as a medicinal herb for many years because of its calming, analgesic, antitumor, and brain cell protection properties. In addition, it is effective in the treatment of women's diseases such as menstrual irregularities, amenorrhea, menopausal syndrome, and postpartum abdominal pain.
Thus, it is sold as a functional food for women's health care in Europe and USA [1-3]. Korean Angelica (Angelica gigas), Japanese Angelica (Angelica acutiloba), and Chinese Angelica (Angelica sinensis) are named based on their places of origin and are known to be different in appearance, major components, and pharmacological effects [4]. Angelica gigas Nakai, a herb which grows for two to three years, exhibits activities such as immune enhancement, antioxidant, antidiabetic, anti-dementia, skin 
whitening, and ultraviolet protection [5-9]. The anti-inflammatory properties of Angelica gigas Nakai assessed using an atopic dermatitis model as well as against curing dry eye syndrome have been recently reported $[10,11]$.

The main components of Angelica gigas Nakai are decursinol, decursin, and decursinol angelate [12]. Decursin and decursinol angelate are structurally isomeric and their properties are very similar because their secondary alcohol groups are esterified to the 3,3-dimethyl acryloyl group and the angeloyl group, respectively [13]. Decursin and decursinol angelate are effective in the treatment of breast, prostate, and lung cancers, whereas decursinol does not directly kill cancer cells [14-16]. However, decursinol was effective in preventing tumor cell proliferation in an in vitro study and is being marketed as "Decursinol-50" owing to its anti-inflammatory and pain-relieving effects [14-18].

In recent studies, metabolic analyses of decursin and decursinol angelate were conducted using the human liver microsome and rodent plasma, which showed that decursinol is produced as a major metabolite of decursin and decursinol angelate [19]. In another study, oxidation and glucuronidation of decursinol were attempted in rats in vitro and in vivo, but no significant metabolites were found [21]. Owing to its remarkable pharmaceutical activity, decursinol has been the subject of significant research in multiple fields, including pharmacokinetic studies; however, metabolic studies of decursinol have not produced any tangible results. In this present study, the metabolism of decursinol in vitro was evaluated in human liver microsomes, and their structures were determined using LC-Q-TOFMS.

\section{EXPERIMENTAL}

\section{Reagents and chemicals}

Decursinol standard was purchased from Chem Faces (Wuhan, China). $\beta$-Nicotinamide adenine dinucleotide phosphate sodium salt hydrate ( $\geq 98 \%$ ), glucose-6-phosphate dehydrogenase from baker's yeast (S. cerevisiae), D-glucose 6phosphate sodium salt, potassium phosphate monobasic, potassium phosphate dibasic, and dimethyl sulfoxide (DMSO) were purchased from Sigma Chemical Co. (St Louis, MO, USA). Mixed-gender pooled 150-donor human liver microsomes were supplied from BD Gentest (MA, USA). HPLC (High performance liquid chromatography) Grade methanol (99.9\%) used for sample preparation was purchased from Duksan Reagent (Korea). HPLC Grade acetonitrile (99.9\%) used for chromatographic mobile phase and sample deproteination was obtained from J.T. Baker (Phillipsburg, NJ, USA). Distilled water was purified using an ultrapure water preparation apparatus (Millipore Elix 3 and Milli-Q Academic Gradient A-10 Bedford, MA, USA).

\section{Evaluation of in vitro metabolism using human liver microsome}

A $50 \mathrm{mM}$ substrate stock solution was prepared by dissolving decursinol in DMSO. $1 \mathrm{M}$ phosphate buffer ( $\mathrm{pH}$ 7.4) was prepared by mixing $1 \mathrm{M}$ potassium phosphate dibasic and 1 $\mathrm{M}$ potassium phosphate monobasic at a volume ratio of 8:2. The NGS (NADPH( $\beta$-Nicotinamide adenine dinucleotide phosphate sodium salt hydrate) generating system)was prepared by adding $500 \mu \mathrm{L}$ of $0.1 \mathrm{M}$ glucose 6-phosphate, $250 \mu \mathrm{L}$ of $10 \mathrm{mg} / \mathrm{mL} \mathrm{NADP}{ }^{+}$, and $1.12 \mu \mathrm{L}$ of glucose 6-phosphatedehydrogenase $\quad(1,000$ units) to a total volume of $751.12 \mu \mathrm{L}$. The microsomes were stored at $-80{ }^{\circ} \mathrm{C}$ and incubated at $37^{\circ} \mathrm{C}$ prior to use.

In in vitro experiments, samples for experiments were prepared in $100 \mu \mathrm{L}$ of $1 \mathrm{M}$ phosphate buffer $(\mathrm{pH} 7.4), 645 \mu \mathrm{L}$ of distilled water, $5 \mu \mathrm{L}$ of substrate stock solution, and $50 \mu \mathrm{L}$ of human liver microsomes, and pre-incubated at $37^{\circ} \mathrm{C}$ for $5 \mathrm{~min}$. Thereafter, the sample was added to a tube containing $200 \mu \mathrm{L}$ of NGS, and another preincubated sample was added to a tube containing $200 \mu \mathrm{L}$ of DW instead of NGS for blank sample. The samples were incubated at $37^{\circ} \mathrm{C}$ for $2 \mathrm{~h}$ in a shaking incubator.

\section{Sample preparation}

To confirm the retention time of decursinol, the decursinol standard was diluted to a concentration of $10 \mathrm{ppm}$ in methanol and kept at $4{ }^{\circ} \mathrm{C}$ until LC-Q-TOF-MS analysis. The samples incubated in vitro were centrifuged at $12,000 \mathrm{~g}$ for $10 \mathrm{~min}$. After centrifugation, the supernatant was transferred to a glass tube and deproteinated by adding $3 \mathrm{~mL}$ of acetonitrile. The samples were centrifuged at $12,000 \times g$ for 10 min, and the acetonitrile layer was placed in another glass tube and concentrated using nitrogen gas. The residue was reconstituted in $100 \mu \mathrm{L}$ of methanol and filtered. The filtered sample was injected into the LC-Q-TOF-MS.

\section{LC-Q-TOF-MS conditions}

The LC-Q-TOF-MS system was composed of an Agilent 1260 Infinity HPLC system (Agilent, Waldbronn, Germany) combined with an Agilent 
6530 Q-TOF mass spectrometer (Agilent Technologies, Santa Clara, CA, USA) with electrospray ionization capability. The chromatographic separation column was $4.6 \times$ $50 \mathrm{~mm}$ I.D. $1.8 \mu \mathrm{m}$ Eclipse XDB-C18 (Agilent Technologies, Foster city, CA, USA), and the column temperature was maintained at $40{ }^{\circ} \mathrm{C}$. The mobile phases used were solvent $A(0.1 \%$ formic acid in water) and solvent $B$ (acetonitrile). The flow rate was set at $0.5 \mathrm{~mL} / \mathrm{min}$. The chromatographic separation was done in a gradient with solvent B \% set to $15 \%$ at 0 to 0.1 $\min , 15$ to $100 \%$ at 0.1 to $7 \mathrm{~min}, 100 \%$ at 7 to 10 $\min , 100$ to $15 \%$ at 10 to $11 \mathrm{~min}$ and $15 \%$ at 11 to $20 \mathrm{~min}$.

For the mass spectrometric parameters used for the mass analysis were as follows: drying gas temperature $200{ }^{\circ} \mathrm{C}$, dry gas flow rate $10 \mathrm{~L} / \mathrm{min}$, nebulizer of $45 \mathrm{psi}$, fragmentor voltage $150 \mathrm{~V}$, capillary $3000 \mathrm{~V}$, skimmer $65 \mathrm{~V}$, Oct RFV $750 \mathrm{~V}$. The mass range was set from $20-1,000 \mathrm{~m} / \mathrm{z}$ and scan rate was 1.00 spectra/sec. Mass measurements for each peak were performed in accordance with an automated calibration delivery system using a calibration solution (calibration solution A, Agilent Technologies) with the internal reference mass at $\mathrm{m} / \mathrm{z} 121.0509$ and 922.0098 .

\section{Statistical analysis}

The ESI probe was operated in the positive mode and data were analyzed using Mass Hunter software (Agilent Technologies). The error ppm was determined by calculating the mass error in parts per million (ppm) from the observed and theoretical $\mathrm{m} / \mathrm{z}$ values. To ensure accuracy, error ppm was analyzed less than 10 .

\section{RESULTS}

To identify the metabolites of decursinol, sample chromatograms with and without NGS were compared and specific peaks in the NGS treated sample chromatogram were identified. Before metabolic identification, the retention time of decursinol in the sample was confirmed by comparing it with that of the decursinol standard. Decursinol and its metabolites were identified mainly in the form of $[\mathrm{M}+\mathrm{H}]^{+}$using the Q-TOFMS spectrum. The extracted ion chromatograms of decursinol and its metabolites analyzed in vitro are shown at Figure 1. All peaks were confirmed using the measured mass. The structures of decursinol metabolites were identified using LCMS and the value of error ppm for determination accuracy was evaluated. (a) (a) blank

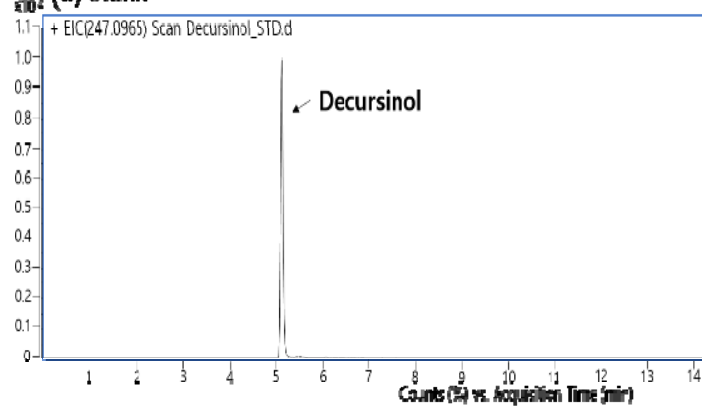

(b) Metabolized sample

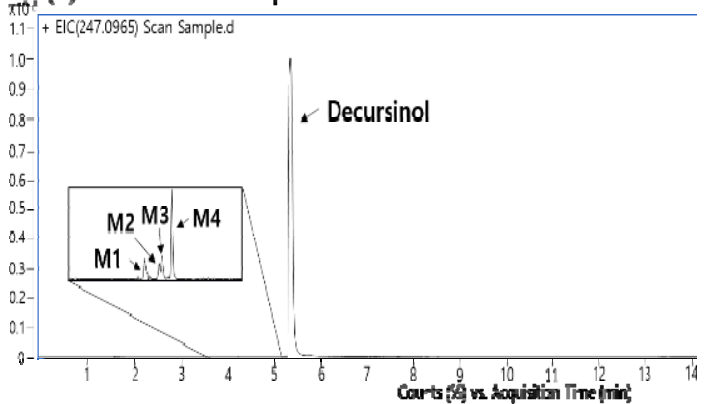

Figure 1: Extracted ion chromatogram of decursinol and its metabolites; (a) blank (b) metabolized sample

The decursinol peak in the samples appeared at the same retention time (5.467 $\mathrm{min}$ ) as that of the decursinol standard. The mass of decursinol and sodium plus ion form identified with LC-Q-TOFMS were $\mathrm{m} / \mathrm{z} 247.0984\left(\mathrm{C}_{14} \mathrm{H}_{15} \mathrm{O}_{4}{ }^{+}\right)$and $\mathrm{m} / \mathrm{z}$ $269.0779\left(\mathrm{C}_{14} \mathrm{H}_{14} \mathrm{O}_{4} \mathrm{Na}^{+}\right)$and the error ppm were $7.78 \mathrm{ppm}$ and $4.01 \mathrm{ppm}$ respectively, which indicates high detection accuracy by LC-Q-TOFMS. The fragments $\mathrm{m} / \mathrm{z}$ of decursinol were $\mathrm{m} / \mathrm{z}$ $229.0878\left(\mathrm{C}_{14} \mathrm{H}_{13} \mathrm{O}_{3}{ }^{+}\right), \mathrm{m} / \mathrm{z} 214.0617\left(\mathrm{C}_{13} \mathrm{H}_{9} \mathrm{O}_{3}{ }^{+}\right)$, and $\mathrm{m} / \mathrm{z} 201.0904\left(\mathrm{C}_{13} \mathrm{H}_{12} \mathrm{O}_{2}{ }^{+}\right)$(Figure 2(a)), respectively, confirming the generation of the same decursinol fragments, as previously observed [19].

The specific peaks detected only in NGS treated samples were regarded as metabolites of decursinol and their MS fragments were interpreted. As a result, a total of four metabolites were identified in the NGS treated samples. These four peaks were hydroxylated structures of decursinol at $\mathrm{m} / \mathrm{z} 263.0915\left(\mathrm{C}_{14} \mathrm{H}_{15} \mathrm{O}_{5}{ }^{+}\right)$with the same molecular weights, and the retention times for M1-M4 were 4.224, 4.704, 4.787, and 5.102 , respectively. All the metabolites eluted faster than decursinol. In addition, the molecular weights for M1-M4 were approximately $\mathrm{m} / \mathrm{z}$ 263.0912-263.0920, and the error values of detected $\mathrm{m} / \mathrm{z}$ were $0.38 \sim 2.29 \mathrm{ppm}$, with low factors suggestive of a high accuracy of mass analysis. 
The common fragments of the four metabolites had an $\mathrm{m} / \mathrm{z} 245.0814\left(\mathrm{C}_{14} \mathrm{H}_{13} \mathrm{O}_{4}{ }^{+}\right)$, with the elimination of $\mathrm{OH}$ group to form a $\mathrm{C}-\mathrm{C}$ double bond. The other fragments used to characterize the structure of the metabolites were identified as $\mathrm{m} / \mathrm{z} \quad 217.0861 \quad\left(\mathrm{C}_{13} \mathrm{H}_{13} \mathrm{O}_{3}{ }^{+}\right), \quad \mathrm{m} / \mathrm{z} \quad 191.0342$ $\left(\mathrm{C}_{14} \mathrm{H}_{15} \mathrm{O}_{5}^{+}\right), \mathrm{m} / \mathrm{z} 175.0393 \quad\left(\mathrm{C}_{10} \mathrm{H}_{7} \mathrm{O}_{3}{ }^{+}\right), \mathrm{m} / \mathrm{z}$ $163.0393\left(\mathrm{C}_{9} \mathrm{H}_{7} \mathrm{O}_{3}{ }^{+}\right)$, and $\mathrm{m} / \mathrm{z} \quad 147.0444$ $\left(\mathrm{C}_{9} \mathrm{H}_{7} \mathrm{O}_{2}{ }^{+}\right)$. Some of them overlapped with the fragments of decursinol, indicating that the fragments were not metabolized.

The fragment ions of $M 1$ were found at $\mathrm{m} / \mathrm{z}$ 175.0384 , indicating that the chromene of decursinol was not metabolized. Therefore, the position where $\mathrm{OH}$ can be bound is the para site or dimethyl of tetrahydropyran.

M2 and M4 showed the same fragment ions at $\mathrm{m} / \mathrm{z} 245.0816$ (M2), m/z 245.0809 (M4), m/z 191.0335 (M2), and $\mathrm{m} / \mathrm{z} 191.0355$ (M4). This indicates that a hydrogen of the $2 \mathrm{H}$-chromen-2one is metabolized to $\mathrm{OH}$. However, the mass spectral information on the fragments was insufficient, and it was difficult to precisely identify the hydroxylated positions of M2 and M4.

M3 was observed at $\mathrm{m} / \mathrm{z} 245.0794, \mathrm{~m} / \mathrm{z}$ 191.0342, and $\mathrm{m} / \mathrm{z}$ 147.0449. Among these ratios, $\mathrm{m} / \mathrm{z} 147.0449$ indicated that the chromen of decursinol was not metabolized, and $\mathrm{m} / \mathrm{z}$ 191.0342 indicated that methyl-chromen of decursinol was hydroxylated. The hydroxylated site of M3 could be identified using the para position of tetrahydropyran. In addition, the $\mathrm{OH}$ position of M3 was identified, and it was concluded that a dimethyl group bound to tetrahydropyran is present at the $\mathrm{OH}$ position of M1. All MS data are summarized in Figure 2 and Table 1.

\section{DISCUSSION}

In previous metabolic study of decursin and decursinol angelate using the human liver microsomes and rodent plasma, several metabolites were identified [19]. Decursin was found to be metabolized to 10 metabolites in vitro, including decursinol. The previously identified metabolites of decursin were one carboxylated, one ester hydrolyzed, one hydrated, one methyl oxidated, three hydroxylated, and three dihydroxylated metabolites, indicating that the metabolism of decursin mainly involved a hydroxylation reaction. In the case of decursinol angelate, a total of 10 metabolites were identified in vitro, including decursinol. Three metabolites formed through hydroxylation and dehydroxylation, two metabolites formed through the epoxidation of hydroxylated forms, one formed through hydration, and one formed through ester hydrolysis. However, the metabolic pathways suggested that none of the compounds were expected to be the metabolites of decursinol. In another study, oxidative and glucuronic metabolism studies were carried out, but no significant metabolites of decursinol have been found [21].
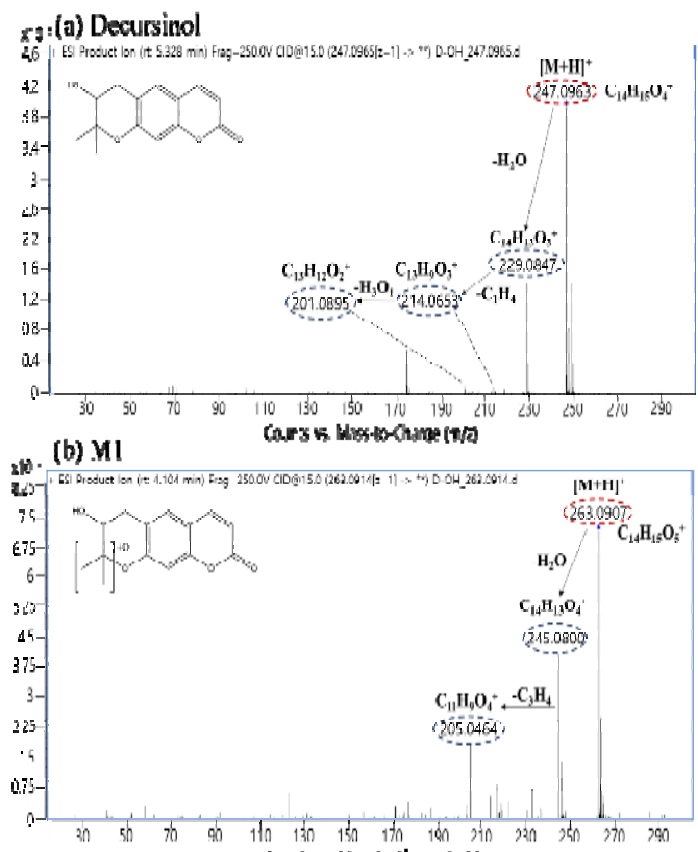

(c) $M 2 \& M 4$
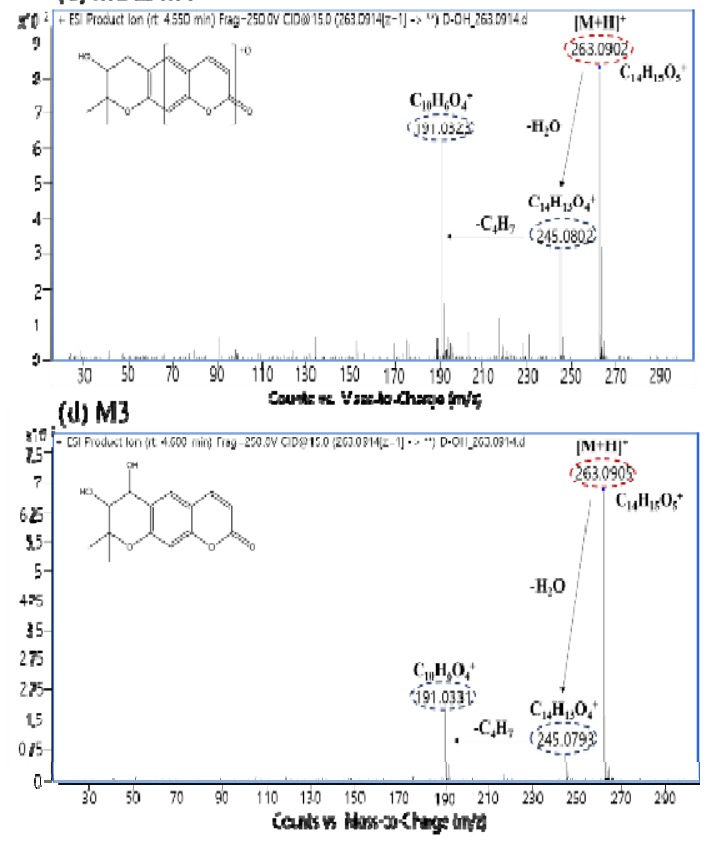

Figure 2: LC-Q-TOF- MS data of decursinol and its metabolites; (a) decursinol (b) M1, (c) M2, (c) M3, (d) M4 
Table 1: LC-Q-TOF- MS and metabolite analysis of decursinol and its metabolites (M1-M4)

\begin{tabular}{lccccccc}
\hline Metabolite & $\begin{array}{c}\text { Formula } \\
{[\mathbf{M + H}]^{+}}\end{array}$ & $\begin{array}{c}\text { RT } \\
(\mathbf{m i n})\end{array}$ & $\begin{array}{c}\text { Theoretical } \\
\text { Mass (Da) }\end{array}$ & $\begin{array}{c}\text { Measured } \\
\text { mass (Da) }\end{array}$ & $\begin{array}{c}\text { Error } \\
(\mathbf{p p m})\end{array}$ & $\begin{array}{c}\text { Metabolic } \\
\text { reaction }\end{array}$ & $\begin{array}{c}\text { m/z of major fragment } \\
\text { fragment ion (Da) }\end{array}$ \\
\hline Decursinol & $\mathrm{C}_{14} \mathrm{H}_{15} \mathrm{O}_{4}{ }^{+}$ & 5.467 & 247.0965 & 247.0984 & 7.78 & Parent & $229.0878,214.0617,201.090447$ \\
\hline M1 & $\mathrm{C}_{14} \mathrm{H}_{15} \mathrm{O}_{5}{ }^{+}$ & 4.224 & 263.0914 & 263.0920 & 2.29 & Hydroxylation & 175.0384 \\
\hline M2 & $\mathrm{C}_{14} \mathrm{H}_{15} \mathrm{O}_{5}{ }^{+}$ & 4.704 & 263.0914 & 263.0915 & 0.38 & Hydroxylation & $245.0816,191.0335$ \\
\hline M3 & $\mathrm{C}_{14} \mathrm{H}_{15} \mathrm{O}_{5}{ }^{+}$ & 4.787 & 263.0914 & 263.0912 & -0.76 & Hydroxylation & $245.0794,191.0342,147.0449$ \\
\hline M4 & $\mathrm{C}_{14} \mathrm{H}_{15} \mathrm{O}_{5}{ }^{+}$ & 5.102 & 263.0914 & 263.0917 & 1.14 & Hydroxylation & $245.0809,191.0355$ \\
\hline
\end{tabular}

Time-of-flight mass spectrometry with high accuracy and high sensitivity made it possible to analyze the decursinol metabolites in this study, and their structures were identified. The four new metabolites detected in the present study were, previously, not included as the metabolites of decursin and decursinol angelate. However, they can be presumed to be metabolites of decursin and decursinol angelate, because decursinol is metabolized from decursin and decursinol angelate. The metabolic pathway of decursinol induced by decursin and decursinol angelate is shown in Figure 3.

These four new metabolites can be used as additional data for further research on decursinol and can be used as significant data for drug interaction analysis. In addition, the metabolism of decursinol and further interpretation was useful in the identification of decursin and decursinol angelate metabolites as well as form a basis for future research on new metabolites of decursin and decursinol angelate.

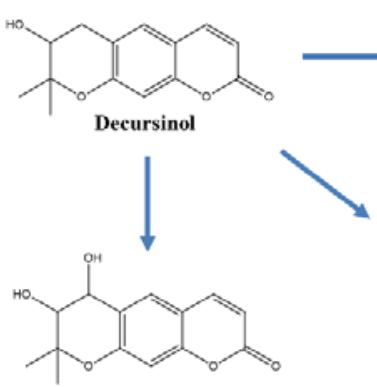

M3

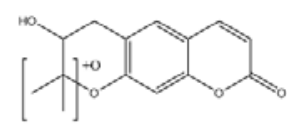

M1

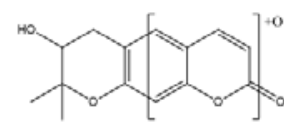

M2 \& M4
Figure 3: Proposed metabolic pathway of decursinol

\section{CONCLUSION}

Decursinol is one of the main bioactive components of Angelica gigas, whose metabolites have not been identified in previous studies. In this study, four new metabolites of decursinol have been identified for the first time in human liver microsomes using high-accuracy LC-Q-TOF-MS. These metabolic data may be useful for food development, as well as studies on Angelica gigas and decursinol, and metabolic enzymes of Angelica gigas. Furthermore, these data can be applied to metabolic and pharmacokinetic studies of decursin and decursinol angelate.

\section{DECLARATIONS}

\section{Acknowledgement}

This research was supported by Korea Basic Science Institute (National research Facilities and Equipment Center) grant funded by the Ministry of Education. (no. 2019R1A6C1010044).

\section{Conflict of interest}

No conflict of interest is associated with this work.

\section{Contribution of authors}

We declare that this work was done by the authors named in this article and all liabilities pertaining to claims relating to the content of this article will be borne by the authors. Jiye $\mathrm{Gu}$ and Jaesung Pyo designed the study and wrote the manuscript. Young Jin Park and Chaeyeong Ahn performed the experimental work. Dayoung Heo carried out a literature study and compiled the data. Yuseong Chung and Kang Min Kim performed a literature survey and analyzed the data. Jae Seon Kang compiled the data. † The first two-named authors contributed equally to this work. The research article was thoroughly read by all the authors before commination for the consideration of publication. These authors contributed equally to this work.

\section{Open Access}

This is an Open Access article that uses a funding model which does not charge readers or their institutions for access and distributed under the terms of the Creative Commons Attribution License (http://creativecommons.org/licenses/by/ 4.0) and the Budapest Open Access Initiative (http://www.budapestopenaccessinitiative.org/rea d), which permit unrestricted use, distribution, and reproduction in any medium, provided the original work is properly credited. 


\section{REFERENCES}

1. Kim GS, Park CG, Jeong TS, Cha SW, Baek NI, Song KS. ACTA (Acyl-CoA:cholesterol Acyltransferase) Inhibitory Effect and Quantification of Pyranocurmarin in Different Parts of Angelica gigas Nakai. J Appl Biol Chem 2009; 52(4): 188-194.

2. Ahn MJ, Lee MK, Kim YC, Sung SH. The simultaneous determination of coumarins in Angelica gigas root by high performance liquid chromatography-diode array detector coupled with electrospray ionization/mass spectrometry. J PHARMACEUT BIOMED 2008; 46: 258-266

3. Kim KM, Kim MJ, Kang JS. Absorption, Distribution, Metabolism, and Excretion of Decursin and Decursinol Angelate from Angelica gigas Nakai. J Microbiol Biotechnol 2009; 19(12): 1569-1572.

4. Sung JS, Bang KH, Park CH, Park CG, Yu HS, Park HW, Seong NS. Discrimination of Angelica Radix Based on Anatomical Characters. Korean J Medicinal Crop Sci 2004; 12(1): 67-72.

5. Han SB, Kim YH, Lee CW, Park SM, Lee HY, Ahn KS, Kim IH, Kim HM. Characteristic immunostimulation by angelan isolated from Angelica gigas Nakai. Immunopharmacology 1998; 40: 39-48.

6. Kang SY, Kim YC. Neuroprotective coumarins from the root of Angelica gigas: Structure-activity relationships. Arch Pharm Res 2007; 30(11): 1368-1373.

7. Kim HM, Kang JS, Park SK, Lee K, Kim JY, Hong JT, Kim YS, Han SB. Antidiabetic activity of angelan Isolated from Angelica gigas Nakai. Arch Pharm Res 2008; 31(11): 1489-1496.

8. Kim JH, Koh SK, Koh HJ, Kwon YA, Kim SH, Kim JG et al. A three-month placebo-controlled clinical trial of INM 176 in the old aged subjects with memory impairment. $J$ KOREAN Neuropsychiatric A 2003; 42(2): 254-262.

9. Zhang J, Wang L, Zhang $Y$, Li L, Tang S, Xing C, Kim $\mathrm{SH}$, Jiang $\mathrm{C}, \mathrm{Lu} \mathrm{J}$. Chemopreventive effect of Korean Angelica root extract on TRAMP carcinogenesis and integrative "omic" profiling of affected neuroendocrine carcinomas. Mol Carcinog 2014; 54: 1567-1583.

10. Ok S, Oh SR, Jung TS, Jeon SO, Jung JW, Ryu DS. Effects of Angelica gigas Nakai as an Anti-Inflammatory Agent in In Vitro and In Vivo Atopic Dermatitis Models.
Evidence-Based Complementary and Alternative Medicine 2008: 12.

11. Lee YJ, Kim KM, Kang JS. Preventive effect of Angelica gigas Nakai extract oral administration on dry eye syndrome. Asian Pac J Trop Med 2018; 11(6): 369-375.

12. Cho MG, Bang JK, Chae YA. Comparison of Volatile Compounds in Plant Parts of Angelica gigas Nakai and A. acutiloba Kitagawa. Korean J. Medicinal Crop Sci 2003; 11(5): 352-357.

13. Kim KM, Jung JY, Hwang SW, Kim MJ, Kang JS. Isolation and Purification of Decursin and Decursinol Angelate in Angelica gigas Naka. J Korean Soc Food Sci Nutr 2009; 38(5): 653-656.

14. Jiang C, Guo J, Wang Z, Xiao B, Lee HJ, Lee EO, Kim $\mathrm{SH}, \mathrm{Lu}$ J. Decursin and decursinol angelate inhibit estrogen-stimulated and estrogen-independent growth and survival of breast cancer cells. Breast Cancer Res 2007; 9: R77.

15. Yim D, Singh RP, Agarwal C, Lee SK, Chi HJ, Agarwal RJ. A novel anticancer agent, decursin, induces $G(1)$ arrest and apoptosis in human prostate carcinomacells. Cancer Res 2005; 65(3): 1035-1044.

16. Lee HJ, Lee EO, Lee JH, Lee JH, Lee KS, Kim KH, Kim $\mathrm{SH}, \mathrm{Lu}$ J. In vivo anti-cancer activity of Korean Angelica gigas and its major pyranocoumarin decursin. Am J Chin Med 2009; 37(1): 127-142.

17. Guo J, Jiang $C$, Wang $Z$, Lee $H J$, Hu $H$, Malewicz $B$, Lee $H J$, Lee JH, Baek NI, Jeong JH, et al. A novel class of pyranocoumarin antiandrogen receptor signaling compounds. Mol Cancer Ther 2007; 6(3): 907-917.

18. Choi SS, Han KJ, Lee JK, Lee HK, Han EJ, Kim DH, Suh $H W$. Antinociceptive mechanisms of orally administered decursinol in the mouse. Life Sciences 2003; 73: 471485.

19. Li L, Zhang JH, Xing CG, Kim SH, Jiang C, Lu J. In Vitro Metabolism of Pyranocoumarin Isomers Decursin and Decursinol Angelate by Liver Microsomes from Man and Rodent. Planta Medica 2013; 79(16): 1536-1544.

20. Yoo HH, Lee MW, Kim YC, Yun CH, Kim DH. Mechanism-Based Inactivation of Cytochrome $P 450$ $2 A 6$ by Decursinol Angelate Isolated from Angelica Gigas. Drug Metab Dispos 2007; 35(10): 1759-1765.

21. Song JS, Chae JW, Lee KR, Lee BH, Choi EJ, Ahn SH, Kwon K, Bae MA. Pharmacokinetic characterization of decursinol derived from Angelica gigas Nakai in rats. Xenobiotica 2011; 41(10): 895-902. 